\title{
A novel instrument for power quality monitoring based in higher-order statistics: a dynamic triggering index for the smart grid
}

\author{
Olivia Florencias-Oliveros, Agustín Agüera-Pérez, Juan-José González-de-la-Rosa, José-Carlos Palomares- \\ Salas, José-María Sierra-Fernández ${ }^{1}$
}

${ }^{1}$ Research Group PAIDI-TIC-168, Computational Instrumentation and Industrial Electronics (ICEI), Spain

\author{
Department of Automation Engineering, Electronics, Architecture and Computer Networks \\ Polytechnic School of Algeciras, University of Cadiz - Av., Ramón Puyol S/N, E11202 (Spain) \\ Phone/Fax number: +0034 956028069, e-mail: olivia.florencias@uca.es, juanjose.delarosa@uca.es, agustin.aguera@uca.es, \\ josecarlos.palomares@uca.es, josemaria.sierra@uca.es
}

\begin{abstract}
This paper presents a novel virtual instrument for PQ assessment, based in higher-order statistics. It implements a new power-quality index, which is thought to trigger the measurement procedure when an electrical fault comes about. The user interfaces include not only the online variance charts but also the skewness and kurtosis graphs, along with hybrid representations of variance versus higher-order statistics. Designed on the basis of 85 signals recorded, $50 \mathrm{~Hz}$ real-time with different disturbances. The instrument validates the PQ procedure during online sessions. The new monitoring strategy activates the analysis of the signal once the PQ index overpassed a predefined threshold. Results show that voltage sags and transients are classified within different clusters, with low uncertainty, using different types of graphs. This flexibility may allow the operator to reduce the subjectivities during the online monitoring session with an optimal computational solution.
\end{abstract}

\section{Key words}

Power quality index, virtual instrument, real-time monitoring, higher-order statistics.

\section{Introduction}

The paradigm of smart grid (SG) tends to incorporate, more and more distributed generation (DG) systems, like wind turbines, photovoltaic modules (PV), fuel cells, etc. The effects of the distributed generation, along with the deregulation of the $\mathrm{SG}$, is increasing the concern on power quality (PQ), as it has a direct impact on utilities and customers, as well as effects on the sinusoidal signal of the power line. The a priori unknown features of the distributed energy resources (DER) introduce non-linear behaviours in loads associated to a variety of PQ disturbances, like voltage sags, swells, interruptions, transients, harmonics, flicker, etc. The anomalies also affect the more sensitive equipment, resetting industrial control systems, producing failures in motors and equipment outages, with the subsequent economic loses ([1],[2],[3]). Several studies in the literature focus the attention on the necessity to improve the effective detection and prevention of the irregularities in the system and the effects in the electrical configuration of customers. The aim is a new strategical standard of PQ criteria that allows designers to optimize future networks.

Thus, many techniques and instruments have been developed for PQ monitoring. In the last few years artificial intelligence and on-line measurements are the main trends in the development of PQ monitoring [4],[5],[6].

The traditional methods for monitoring PQ anomalies, analyzed the evolution of the spectral component (FFT), the frequency's change in the time domain (wavelets transforms) and the total harmonic distortion (THD) index ([7], [8]). These techniques are only based in the static spectrum, and cannot be applied on non-stationary signals, like transients. In addition, noise degrades the effectiveness of these second-order techniques. Recent studies concluded that a better characterization of symmetry and shape of the waveform are possible via the 3erd and 4th higher-order statistics (HOS), that also account for the nonlinear assessment of the signals [9], [10].

Some studies have developed the cluster analysis of 4th-order estimates that inform about the duration and intensity of PQ transients. They use higher order cumulants as the feature parameter ([11], [12]), and quadratic classifiers as the classification method [12]. This techniques can be applied on data generated by the sensors in the SG [13].

Nevertheless, the implementation of HOS algorithms in intuitive graphical user interfaces is not easy, as it demands the operator a higher degree of scientific knowledge. In fact, the traditional displays and graphs have been improved with additional information, e.g. phasors and a set of energy related. In this sense, it would be useful to develop a new power quality index which include information of the PQ events regarding the HOS measurements [14].

This paper introduces a new HOS-based PQ index that triggers the $\mathrm{PQ}$ measurement session when an electrical anomaly bumps into the system and proposed 
subsequent analysis of the signal. Wireless-based in conception, it has been designed under the norms of the European Standard EN501690:2007 [15] and the testing and measurement techniques are based in the Spanish Standard for PQ measurement methods [16].

The quality of the voltage range allows $\pm 10 \%$ of voltage variation depending on the influence of the measuring point where the system voltage is monitored, the instant at which the signal is disturbed, the type of disturbance, the influence of the internal configuration of the user's network and the connected equipment.

All data records of all monitored signals analyses are real disturbances obtained from the network of the University of Cádiz.

The document has structured as follows. Section 2 introduces the new PQ index; Section 3, the PQ experimental strategy, Section 4, the virtual instrument and results. Finally, in the Section 5, the conclusions are drawn.

\section{The New Power Quality Index}

A novel virtual instrument for power quality analyses has been implemented in a computational platform, in order to monitoring electrical power signals in real time.

The instrument, based in higher-order statistics, indicated the global signal stability and executed a new Power Quality Index (PQ index) (1), capable to detect the signal anomalies in the power line and trigger a classification analysis focused on the degree of the events.

The estimator analyses a sinusoidal signal through a sliding window, considering the absolute values of the statistics evaluated in the time domain. Signals of $50-\mathrm{Hz}$ with a sampling frequency of $20 \mathrm{kHz}$ are acquired from the power line. The sliding window sweeps the whole data register, each copes with one cycle, and no overlapped (Fig.1) (2). A deeper description of this technique can be found in [5], [9].

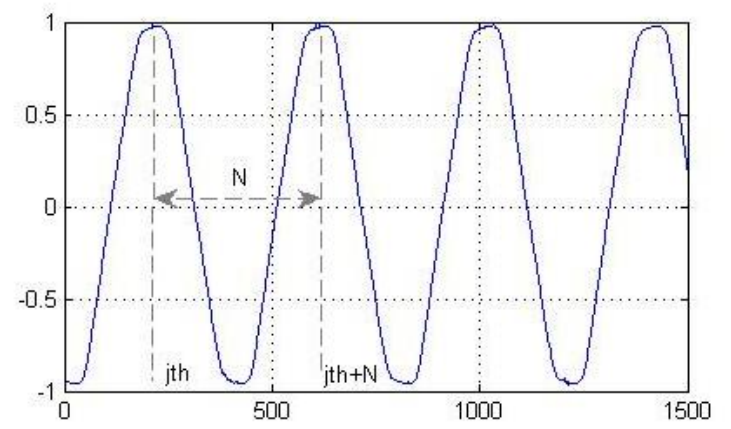

Fig.1 Representation of the sliding window, in a $50 \mathrm{~Hz}$ power signal recorded with a sampling frequency of $20 \mathrm{kHz}(400$ samples per cycle). The number of data-cycles is represented by $\mathrm{N}$ samples and $\mathrm{j}+\mathrm{N}$ samples the sequence.

\section{A. Power Quality Index and HOS}

The procedure of the analysis is based on the comparison of the values of variance (var), skewness (skew) and kurtosis (kurt) associated to each cycle, versus the theoretical values of a healthy signal (3). As established in the previously cited works, a healthy signal (i.e. a 50$\mathrm{Hz}$ sinusoidal signal) would be characterized by theoretical values variance $(0,5)$, skewness $(0)$, kurtosis $(1,5)$. One of the abilities of HOS is to represent the data https://doi.org/10.24084/repqj15.212 set in a new feature space in which the probability to distinguish signals properties is higher than in the original space. In that sense, an effective comparison of HOS are based on the PQ index to each cycle. The PQ values estimated from the sampled signal are always absolute values. Thus, the proposed PQ index for the jthcycle can be defined as the following equation:

$$
\begin{aligned}
& P Q_{j}=\operatorname{abs}\left[0.5-\operatorname{var}\left(X_{j}, \ldots X_{j+N}\right)\right] \\
& +\operatorname{abs}\left[\mathrm{O}-\operatorname{sken}\left(X_{j}, \ldots X_{j+N}\right)\right] \\
& +\operatorname{abs}\left[1.5-\operatorname{kurt}\left(X_{j}, \ldots X_{j+N}\right)\right]
\end{aligned}
$$

The sequence of each number of cycles $(\mathrm{j} t h-\mathrm{j} t h+N)$, represented:

$$
\left(X_{j}, \ldots X_{j+N}\right)=X_{j}^{N}
$$

Finally, the PQ index is expressed:

$$
\begin{aligned}
& P Q_{j}^{N}=a b s\left\lfloor 0,5-\operatorname{var}\left(X_{j}^{N}\right)\right\rfloor+\operatorname{abs}\left\lfloor\operatorname{sken}\left(X_{j}^{N}\right)\right\rfloor \\
& +a b s\left[1,5-\operatorname{kurt}\left(X_{j}^{N}\right)\right]
\end{aligned}
$$

\section{Power Quality experimental strategy}

The PQ experimental strategy implemented the analysis steps according the organigram of the Fig.2 (Data Acquisition-PQ Evaluation-Classification Analysis).

First, the Data Acquisition session are based in HOS analysis. A healthy signal would obtain a PQ index of 0 . This is an ideal case and all real-life signals contain a certain level of perturbation, thereby producing PQ indexes higher than 0 . In order to determine if the power line signal contains a relevant disturbance, a threshold must be stablished. In the proposed procedure, (PQ Evaluation) the classification analysis are triggered once the PQ index overpasses a specified threshold, which indicates a fault is relevant. The Classification Analysis is performed in three steps: Data Collection, Graph Representation and Cluster Analysis.

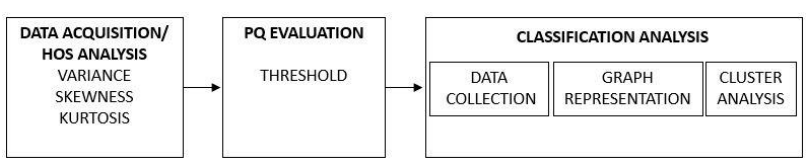

Fig. 2. PQ experimental strategy following the procedure of PQ analysis based in the HOS, threshold data collected and a classification analysis of the estimators.

The cluster analysis are proposed by previously cited works ([11], [12]), in our case, we focused in the HOS average. The PQ Data Collected are classified through the cluster analysis and the characteristically deviation of the average of the corresponding HOS values that help to define a signal space (cluster). In that sense, the battery of signals, sags and transients, are analysed in the virtual instrument (Fig.3-5). In the sample analysed we found results most representative when comparing 2th-order vs. 3 th-order estimates (variance vs. skewness) and 2th-order vs. 4th-order estimates (variance vs. kurtosis), instead of compared 2th-order vs. 3 th-order estimates (skewness vs. kurtosis). In the first two cases (Fig.3 and 4), we found a cluster or a tendency in data. The indicators demonstrate a propensity of create groups of points, or a behaviour RE\&PQJ, Vol.1, No.15, April 2017 
characteristic, evident most of cases, during the transient disturbances. In the third case (Fig. 5), it is quite difficult to compare them and have successful results about data clusters because the skewness and kurtosis data creates a big intersection area. Nevertheless it is confirmed that sags have more accurate $3 \mathrm{er}$ and $4^{\text {th- }}$ order estimators face to the instability of the values of the transients.

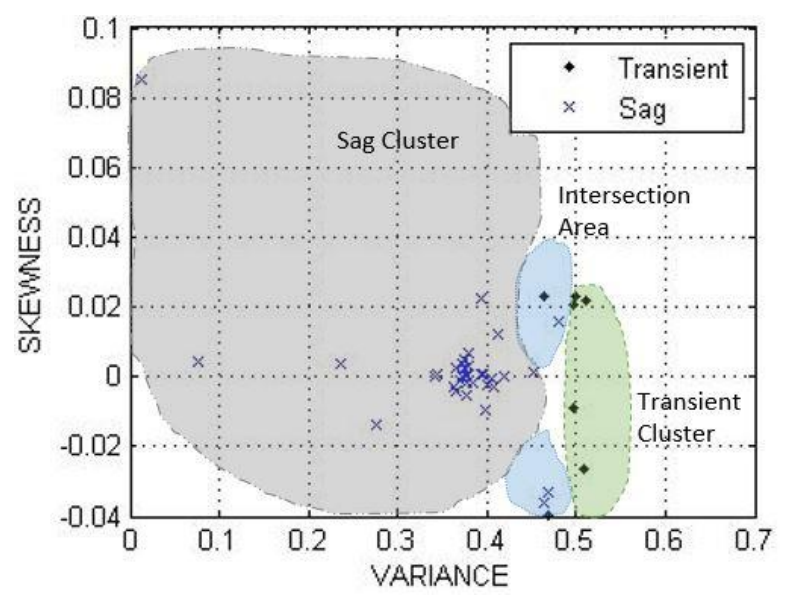

Fig. 3. Sags and Transients Cluster Tendency (average of variance vs. skewness values), from the PQ instrument panel.

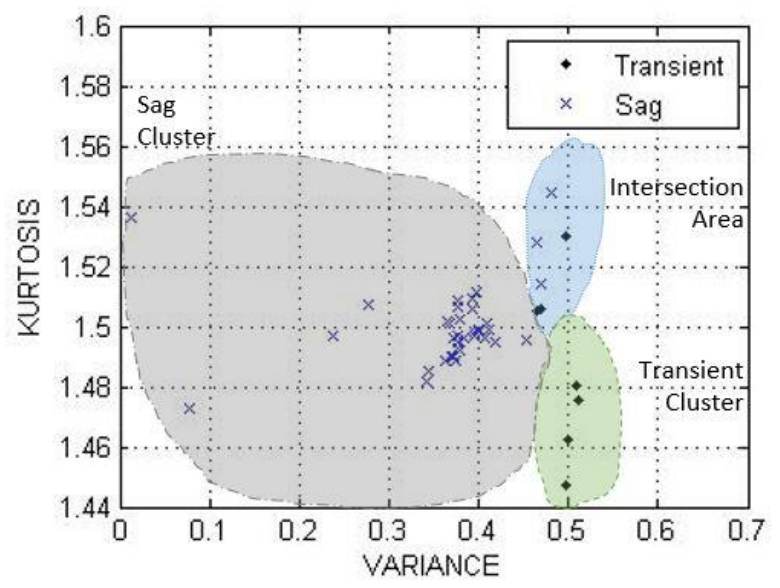

Fig. 4 Sags and Transients Cluster Tendency (average variance vs. kurtosis values), from the PQ instrument panel.

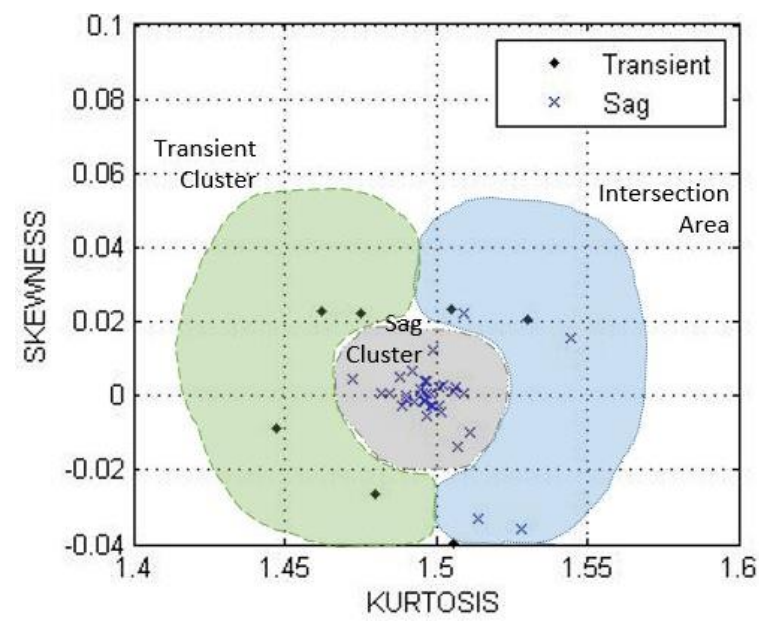

Fig. 5 Intersection area, (average skewness vs. kurtosis values), from the PQ instrument panel.
The PQ experimental strategy (Subsection 3.A) was performed means the validated analysis and developed in the virtual instruments, that accelerate the monitoring of power line and survey the behaviour of cumulates, arriving at similar conclusions. The comparative analysis reach the approach of classified sags and transients into different clusters data.

\section{A. Power Quality in the real-time analysis}

Following the above criteria, and in order to evaluate the PQ index, a group of real signals have been analysed (60 signals presented different sags and 25 transients) at a sampling frequency of $20 \mathrm{kHz}$ (400 samples per cycle). All the signals are events collected from the network of the University of Cádiz, in the past three years. The $41 \%$ presented an rms voltage variation of $80 \%$ and a $43 \%$ with an rms voltage variation of $90 \%$ too.

Transient's disturbances are detected when PQ are superior of 0.02 values. In addition, Sags disturbances are detected when PQ have values superior of 0.05 . In the real-time analysis, it is common to obtain PQ values 0.026 which indicates that the signal under test is not perfect (healthy signal, $\mathrm{PQ}=0$ ). As a result $\mathrm{PQ}=0,02$ values are the PQ flour (PQ values of the network in normal conditions) measured by the instrument.

In addition, according to the analysis the PQ Threshold are considered as twice the value of the PQ flour $(\mathrm{PQ}>=0,05)$ as an empirical conclusion. Finally the PQ values more nearly of 1 points, could represent diverse causes of relevant disturbances.

A graphical description of the real-time analysis in two signals (first Sag 60-70\% and the second one transient) is shown in Figure 6. The experimental strategy previously described are implemented in the first two columns. The column Acquisition Data, characterized the HOS theoretical values of a signal in the time domain, with emphasis in the cycles perturbed. The effectiveness of the PQ Evaluation in monitoring signals are clearly visible with the deviation of the PQ characteristic values on the perturbed cycles (PQ vs. data index). The goal of the third column is the cclassification analysis procedure. In both cases, the Classification Analysis, collected the disturbance of sags and transient's data, the average of them is represented in the previous clusters analyzed (Fig.3-5). The Sag Cluster detected the average of electrical perturbation and the Transient Cluster too. This means that Variance vs. Skewness and Variance vs. Kurtosis graphs are effective methods to discriminate between these two PQ events.

This series of signals captured are an example of the PQ based in HOS operation monitoring implemented in the instrument and gives additional information to improve the instrument measurement capacity, and the subsequent analysis criteria of the signal. The PQ index displayed the process capacity of the signals with a high certitude level. The procedure validates the implementation of a computational solution on PQ real-time analysis.

\section{The novel virtual instrument and results}

The instrument configuration are supported by an organigram measurement, according with the Measurement Methodology proposed by the UNE-EN 61000-4-30 (Fig. 7). The aim is, design an instrument (S RE\&PQJ, Vol.1, No.15, April 2017 
Classification) that measure the power line (CA 230V, $50 \mathrm{~Hz}$ ) in the corresponding intervals.

The hardware based in a HAMEG Instrument Differential probe, HZ 115 amplifies the signal from the consumer power line (measuring transducer). The data acquisition system are possible through an Ethernet connection with the chassis NIcDAQ ${ }^{\mathrm{TM}}$ 9188, National Instruments technology that supported an analogue input module, NI 9225 from the $\mathrm{C}$ series (measuring devices).

The chassis connected to ASUS X554L portable computer via Ethernet connection. The virtual interface is designed in the software LabVIEW ${ }^{\mathrm{TM}}$ (2014) that function as evaluation unit and consecutive analysis of the data acquisition.

\section{A. Instrument Interface}

The instrument's interface are based in panels (Fig.8, 9) and present two operating PQ Control Modes, see Fig. 8.

1) Mode 1. Characterization of the Power Line. Coherent with the normative, capable of monitoring voltage, harmonics, inter harmonics and network imbalance. Implement an elementary window of 10 cycles $(10 \mathrm{~T})$, with a critical time interval measurement of 15 blocks of $10 \mathrm{~T}(150 \mathrm{~T})$. The measurement procedure, analyses the elementary windows in continuous (UNE-EN 61000-4-30).

2) Mode 2. Detection Transients. It proposes an alternative procedure to the normative, based on the sliding window method ([5], [9]) with the aim of measuring each cycle and obtain measurement results more reliable.
There are two PQ values according to the mode setting. The Detection Transients Mode (Fig.8) shows the PQ index on real time, while the Characterization Mode performs an average of the elementary window, as if it were a filter for the power quality (PQ values $10 \mathrm{~T}$, (150 $\mathrm{T})$ ), detecting less PQ events.

The main panel "PQ Control" (Fig.8) allows configuring the information for the work session: number of the session, network analysed, the name of the operator, the file path for signal storage for the posterior analysis. Also, the normalized signal acquisition is present and the PQ indexes tendency. The session is controlled and any time possible abort. The other new panel is "PQ Threshold" (Fig. 9) give to the operator the option of monitoring to PQ behaviour: real-time and characteristic according to the mode, PQ value, HOS disturbance tendency, PQ Flour detection and PQ Threshold Alarm. The input of the instrument automatically computes the degree of disturbance of a signal on a power line, measuring the typical PQ of the network. The Wireless Monitoring Network Procedure, allows you to monitor the network and maintain active working session through LabVIEW (Fig.7).

\section{A. The $P Q$ classification results}

Additionally, using the PQ it is possible to realise a Classification Analysis: set an intuitive threshold for the monitoring sessions that permits to design leds alarms, bottoms stops, saves graphics of HOS behaviour; update the online session, save a cluster analysis and more control options available (Fig.8, 9). There are other panels developed that are commons in similar virtual instruments, it is the case of "HOS", measuring the statistics on real time, showing the max and min values in a range of data sample.

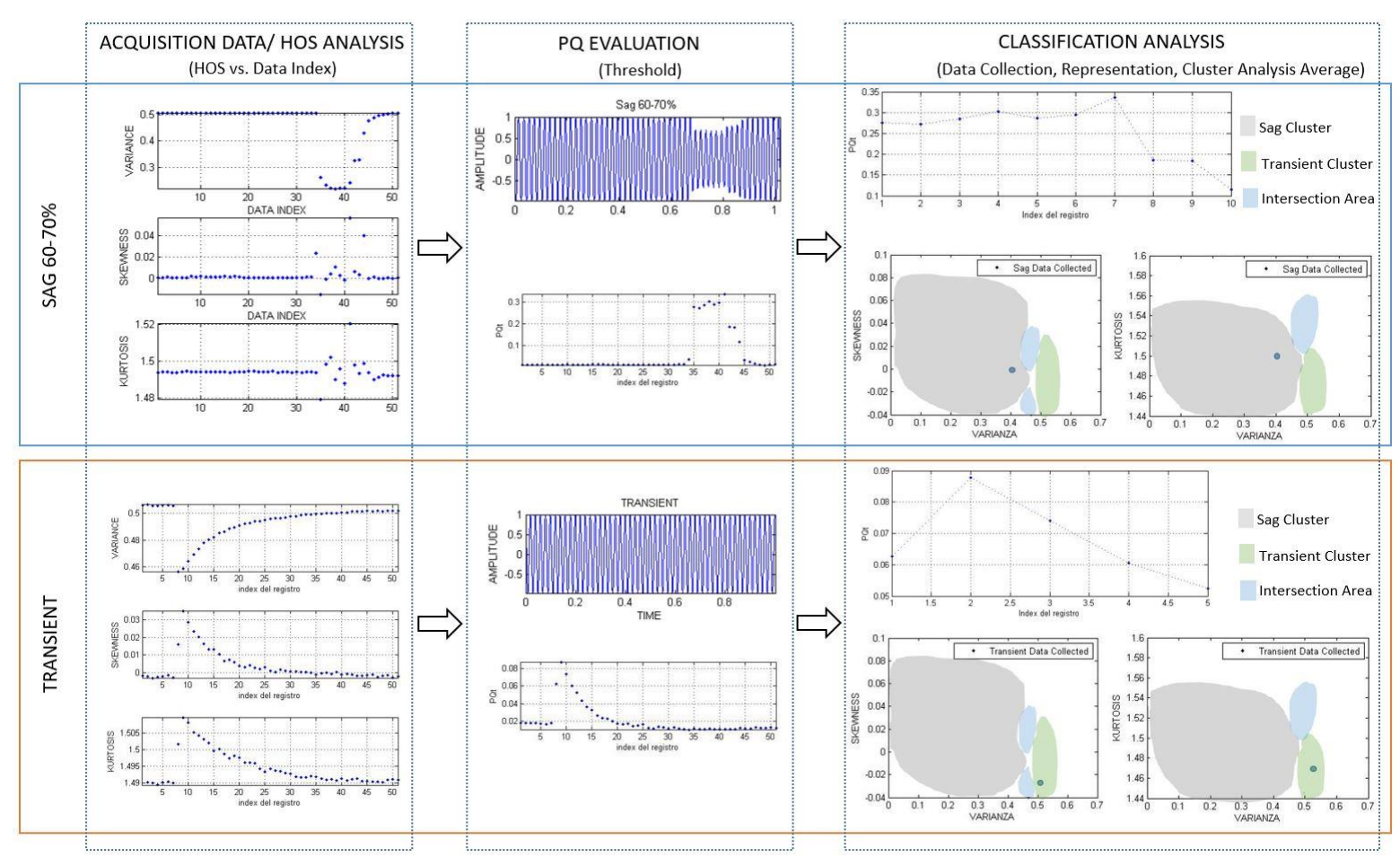

Fig. 6 Representation of the PQ experimental strategy in the real-time analysis of two- signals disturbed, $50 \mathrm{~Hz}$, sampling frequency of 20 $\mathrm{kHz}$ (400 samples per cycle). The first, sag $10 \%$, and the second with a transient's disturbance. The establish methodology different analysis steps with the objective of classified the behaviour of disturbances. The signal has 50 PQ index according to the samples. When a sag occurs, the PQ index helps to determine the subsequent behaviour, using then, is possible to observe that the signal show a tendency

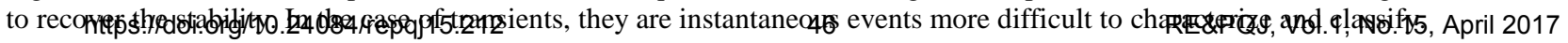




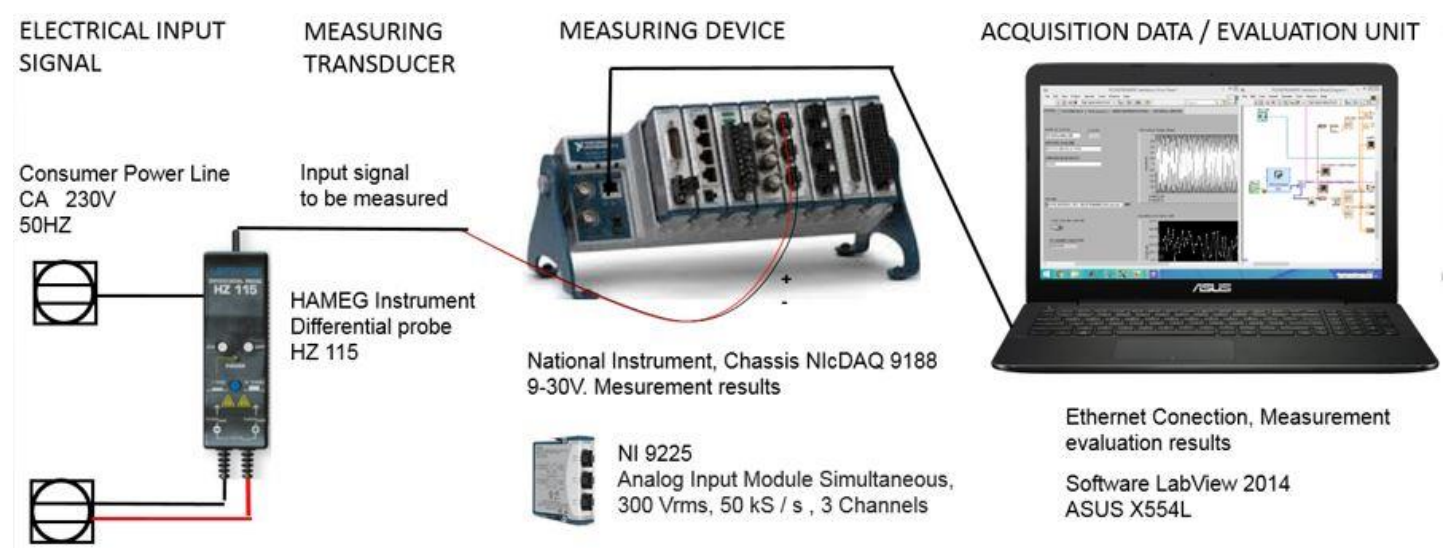

Fig. 7. The virtual Instrument Configuration guarantee the measurement traceability of the devices. The hardware consists in certificates instruments, such as HAMEG and National Instruments technology. The interface allows on-line analyzing graphs, process signals on real-time and report incidents.

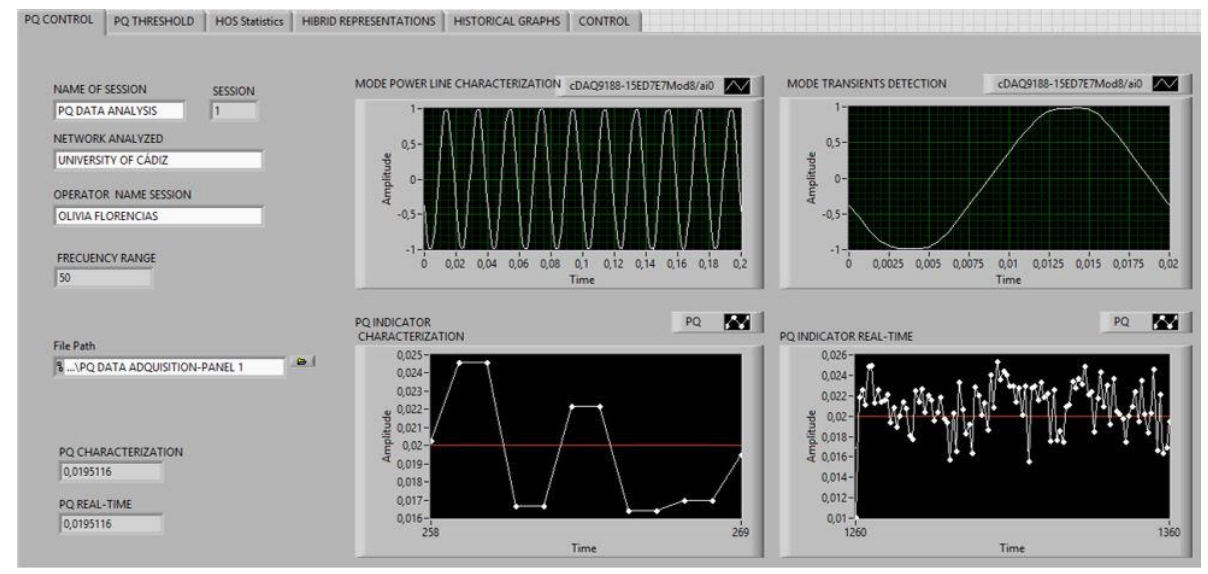

Fig. 8. PQ Control Panel. At the left, allows the operator to configurate the session and save the information. The rest of the panel focuses on presenting the different operating models: Mode Characterization of the Power Line (left) and Mode Detection Transients (right), following the PQ indexes tendency in both modes. The horizontal line in the PQ pannels (red) represent the PQ flour=0,02.

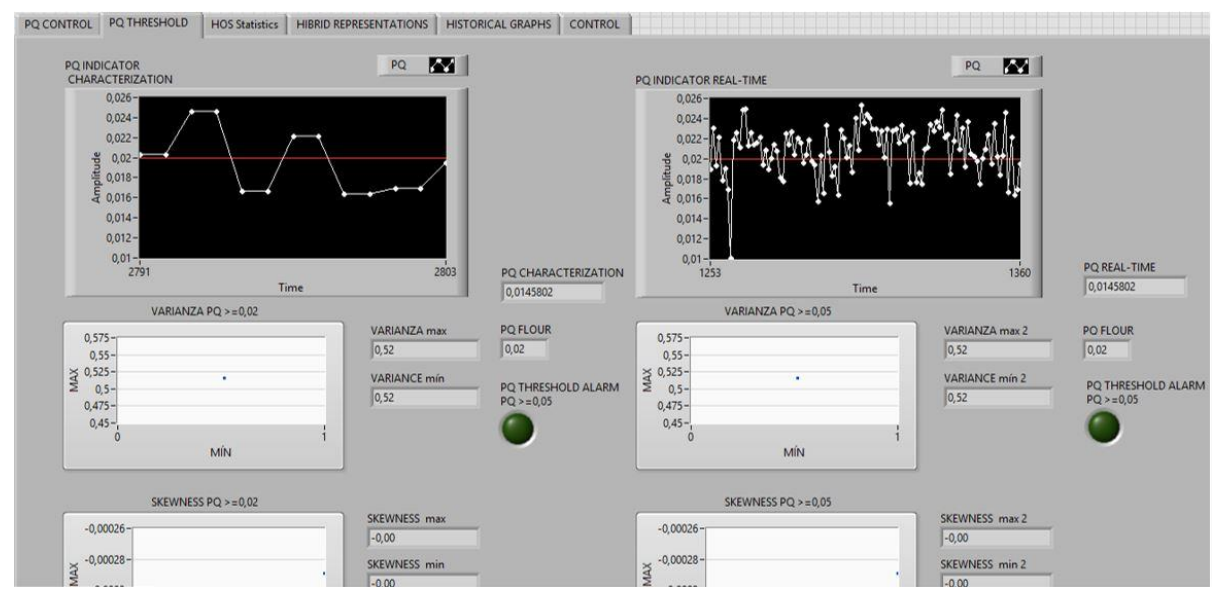

Fig. 9. PQ Threshold Panel monitored the PQ behaviour in both modes, Power Line and Transients Detection. The operator selected the conventional configuration or an specific one in function of variables to monitoring, threshold alarms, and the data needed to save. The Mode Detection is more efective to detect PQ events and measure more accurately the PQ instances values, as is possible to observe when overpassed the PQ flour $(\mathrm{PQ}=0,02)$ and the $\mathrm{PQ}$ Threshold Alarm $(\mathrm{PQ}>=0,05)$ is setting.

Also, historical graphs represent, based on histograms and collected data, a useful to generate automatically reports.

In addition, an innovation in the virtual instrument it is the cluster analysis or "hybrid representations", of several data acquisition on real time disturbed area. In this case, the hybrid representations correspond with theoretical XY graph, using individual statistics values vs. other statistics of signal: variance vs. skewness, variance vs. kurtosis and kurtosis vs. variance (See Fig. 3, 4, 5). The cluster method included other graphs in the virtual instrument as diverse maximum and minimum graph corresponding with the real value of individual statistics according to 
the behaviour of a number of cycles data (limits): variance ( $\max$ vs. min), skewness (max vs. min), kurtosis (max vs. min). The comparison of the limits offers the possibility to adapt the real session with the empirical experience, and the introduction of new measurement alternatives.

\section{Conclusions}

This paper has introduced a novel virtual instrument that implements a new Power Quality Index, tested with a battery of real-life signals. The PQ index has a twofold purpose: to set a threshold for the power signal that in turn enables the PQ analysis associated to the concrete location in the network. Finally, the experimental strategy with real-life signals demonstrates the validity of the procedure to measure the stability of the signal in the time domain through the proposed PQ index.

The new virtual instrument validates some new criteria's introduced in the experimental strategy, the HOS approach helps in the spatial representation and the classification analysis of signal behaviour. The PQ Threshold, additionally detected each time a minimum of level disturbances. It is a useful element for subsequent analyses, considering the present and the collected data previously. The evaluative and predictive methodology of the Classification Analysis streamlines the capture process, reduces storage space of the data register, requiring less samples to monitoring the voltage signal, and obtain effective conclusive results over the traditional analysis (HOS and wavelets).

The instrument proves to be a powerful analysis tool with an efficient procedure of representation, in order to obtain results more accurate on time, and operative sessions more adapted to ordinary users. The Mode of Transients, measure more specifically the failures of power line that information is useful in order to predict better the degradation in all class of equipment. Outcomes of the online monitoring sessions confirmed the theoretical approach of cluster tendency, that found in different graphs analysed. Two clusters (sags and transients) are clearly depicted and the intersection area too. Actually, there are no conclusive results if these strategies of clustering, could be implemented on others disturbances analysed, in order to characterize them.

Finally, the solutions are more than opportune at a professional level as enterprise technical distributions, the power instrumentation marker and other potential clients, in order to introduce new index in equipment's control and monitoring the quality of the power delivered to the final consumer. Measurement results more and more accurate are demands for taking decisions, diagnostics purposes, meters and reliability analysis. The new PQ criteria's exposed a revealing role, a novel index of power quality in the improvement of the standard, TC-39 - Measurements in Power Systems. The aim is add new perspectives to management of the SG, the DER and achieve alternative systems more inclusive in the global network.

\section{Acknowledgement}

The authors thank the Spanish Ministry of Economy and Competitiveness for funding the research project
TEC2013-47316-C3-2-P, ADvanced TEChniques for Power Quality Reliability analysis.

\section{References}

[1] M. H. J. Bollen, S. Bahramirad, and A. Khodaei, "Is there a place for power quality in the smart grid?," Proc. Int. Conf. Harmon. Qual. Power, ICHQP, pp. 713-717, 2014.

[2] M. Farhoodnea, A. Mohamed, H. Shareef, and H. Zayandehroodi, "Power Quality Analysis of GridConnected Photovoltaic Systems in Distribution Networks," Przeglad Elektrotechniczny, no. 2, pp. 208-213, 2013.

[3] C. Muscas, "Power quality monitoring in modern electric distribution systems," IEEE Instrum. Meas. Mag., vol. 13, no. 5, pp. 19-27, 2010.

[4] I. Monedero, C. Leon, and J. Ropero, "Classification of Electrical Disturbances in Real Time Using Nueral Networks," IEEE Trans. Power Deliv., pp. 1-9, 2007.

[5] J. José, G. De, A. Agüera-Pérez, J. C. Palomares-Salas, J. M. Sierra-Fernández, and A. Moreno-Muñoz, "A novel virtual instrument for power quality surveillance based in higher-order statistics and case-based reasoning," Mesurement, vol. 45, pp. 1824-1835, 2012.

[6] S. S. S. R. Depuru, L. Wang, and V. Devabhaktuni, "Smart meters for power grid: Challenges, issues, advantages and status," Renew. Sustain. Energy Rev., vol. 15, no. 6, pp. 2736-2742, 2011.

[7] M. H. J. Bollen, I. Y. H. Gu, P. G. V Axelberg, and E. Styvaktakis, "Classification of underlying causes of power quality disturbances: Deterministic versus statistical methods," EURASIP J. Adv. Signal Process., vol. 2007, 2007.

[8] L. Angrisani, P. Daponte, and M. D\&apos;Apuzzo, "Wavelet network-based detection and classification of transients," IEEE Trans. Instrum. Meas., vol. 50, no. 5, pp. 1425-1435, 2001.

[9] J. C. As, J. José, G. De, J. M. Sierra-Fernández, D. AyoraSedeño, and A. Moreno-Muñoz, "Characterization of electrical sags and swells using higher-order statistical estimators q," Measurement, vol. 44, pp. 1453-1460, 2011.

[10] M. V. Ribeiro, C. A. G. Marques, C. A. Duque, A. S. Cerqueira, and J. L. R. Pereira, "Detection of disturbances in voltage signals for power quality analysis using HOS," EURASIP J. Adv. Signal Process., vol. 2007, 2007.

[11] J. J. G. De La Rosa, A. Moreno, and C. G. Puntonet, “A practical review on higher-order statistics interpretation. Application to electrical transients characterization," ISPA 2007 - Proc. 5th Int. Symp. Image Signal Process. Anal., pp. 224-229, 2007.

[12] Ö. "N. Gerek and D. G. Ece, "Power-quality event analysis using higher order cumulants and quadratic classifiers," IEEE Trans. Power Deliv., vol. 21, no. 2, pp. 883-889, 2006.

[13] K. Zhou, C. Fu, and S. Yang, "Big data driven smart energy management: From big data to big insights," Renew. Sustain. Energy Rev., vol. 56, pp. 215-225, 2016.

[14] 1159-1995 IEEE Std, IEEE Recommended Practice for Monitoring Electric Power Quality. 1995.

[15] UNE-EN 50160: "Voltage characteristics of electricity supplied by public distribution networks", 2008, [Online] Available: http://www.aenor.es/.

[16] UNE-EN 61000-4-30: "Electromagnetic compatibility (EMC). Part 4-30: Testing and measurement techniques Power quality measurement methods", 2009. 